
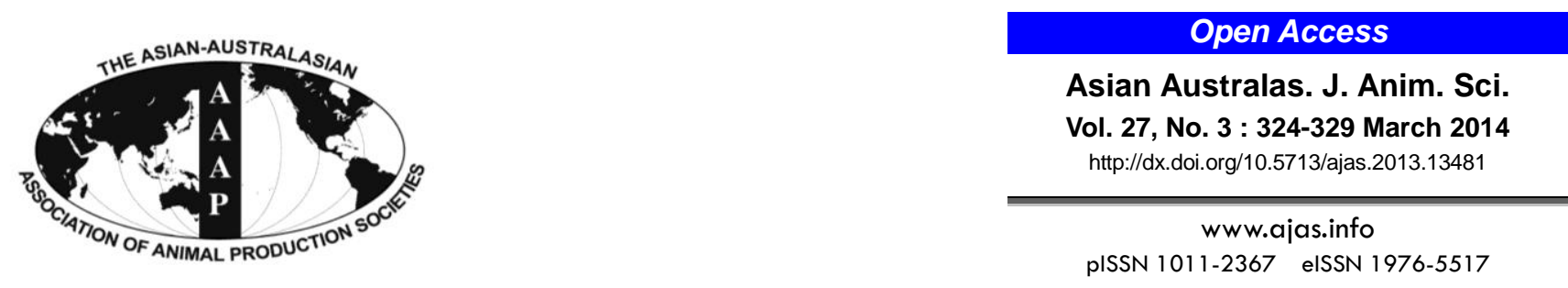

\title{
Production of Mutated Porcine Embryos Using Zinc Finger Nucleases and a Reporter-based Cell Enrichment System
}

\author{
Ok Jae Koo ${ }^{1, a}$, Sol Ji Park ${ }^{2, a}$, Choongil Lee ${ }^{3, a}$, Jung Taek Kang', Sujin Kim², Joon Ho Moon², \\ Ji Yei Choi ${ }^{2}$, Hyojin Kim ${ }^{3}$, Goo Jang ${ }^{2,4}$, Jin-Soo Kim ${ }^{3}$, Seokjoong Kim ${ }^{5}, *$, and Byeong-Chun Lee ${ }^{2, *}$ \\ ${ }^{1}$ Laboratory Animal Research Center, Samsung Biomedical Research Institute, Gyeonggi-do 440-746, Korea
}

\begin{abstract}
To facilitate the construction of genetically-modified pigs, we produced cloned embryos derived from porcine fibroblasts transfected with a pair of engineered zinc finger nuclease (ZFN) plasmids to create targeted mutations and enriched using a reporter plasmid system. The reporter expresses RFP and eGFP simultaneously when ZFN-mediated site-specific mutations occur. Thus, double positive cells $\left(\mathrm{RFP}^{+} / \mathrm{eGFP}^{+}\right)$were selected and used for somatic cell nuclear transfer. Two types of reporter based enrichment systems were used in this study; the cloned embryos derived from cells enriched using a magnetic sorting-based system showed better developmental competence than did those derived from cells enriched by flow cytometry. Mutated sequences, such as insertions, deletions, or substitutions, together with the wild-type sequence, were found in the cloned porcine blastocysts. Therefore, genetic mutations can be achieved in cloned porcine embryos reconstructed with ZFN-treated cells that were enriched by a reporter-based system. (Key Words: Knockout Pig, Reporter Based Enrichment, Somatic Cell Nuclear Transfer, Zinc Finger Nuclease)
\end{abstract}

\section{INTRODUCTION}

Pigs are a useful experimental animal for biomedical research because of their anatomical and physiological similarities with humans (Smith and Swindle, 2006) and relatively high production efficiency (Koo et al., 2012). For these reasons, various transgenic pigs have been generated

\footnotetext{
* Corresponding Authors: Byeong-Chun Lee. Tel: +82-2-8801269, Fax: +82-2-873-1269, E-mail: bclee@ snu.ac.kr / Seokjoong Kim. Tel: +82-2-873-8170, Fax: +82-2-873-8169, E-mail: sj.kim@toolgen.com

${ }^{2}$ Department of Theriogenology and Biotechnology, College of Veterinary Medicine, Seoul National University, Seoul 151-742, Korea.

${ }^{3}$ National Creative Research Initiatives Center for Genome Engineering, Department of Chemistry, Seoul National University, Seoul 151-742, Korea.

${ }^{4}$ Emergence Center for Food-Medicine Personalized Therapy System, Advanced Institutes of Convergence Technology, Seoul National University, Gyeonggi-do 443-270, Korea.

${ }^{5}$ ToolGen Inc., Seoul 153-783, Korea.

${ }^{a}$ These authors contributed equally.

Submitted Aug. 7, 2013; Accepted Sept. 25, 2013; Revised Oct. 29, 2013
}

since the middle of the 1980s (Whyte and Prather, 2011). However, production of knockout or gene targeted pigs was extremely rare until very recently because of very low efficiency of homologous recombination in somatic cells and a lack of appropriate technologies, such as embryonic stem cells, in this species. Lately, pioneering work using zinc finger nucleases (ZFNs) to produce knockout rats (Geurts et al., 2009) enabled us to perform a new approach for producing knockout mammals without homologous recombination or embryonic stem cells. ZFNs are engineered proteins composed of the FokI endonuclease domain linked to a DNA-binding zinc finger protein domain. Pairs of the ZFNs efficiently generate sequencespecific DNA double-strand breaks on a chromosome; consequently, targeted mutations can be introduced into cells of interest (Kim et al., 2009; Kim et al., 2011). Since Watanabe et al. (2010) first showed that the ZFN system can be used in porcine cells, several knockout pigs have been generated using ZFNs and somatic cell nuclear transfer (SCNT) techniques.

However, the protocols developed in the previous studies cannot be used as a general application for 
producing knockout pigs with ZFNs. Most of the previous reports screened for the presence of $\mathrm{ZFN}$-mediated mutations in donor cells by detecting loss of a specific surface molecule (Hauschild et al., 2011; Li et al., 2013; Lutz et al., 2013) or ectopic marker gene expression (Whyte et al., 2011) affected by the mutation. For example, a specific surface molecule, alpha 1,3-galactose, is only available to detect mutations of alpha 1,3 galactosyltransferase and related genes. Also, ectopic marker gene expression, such as eGFP or luciferase, is necessary to generate the cell lines which have the gene sequences prior to gene targeting. Unfortunately, most of the other mutant cells generated by $\mathrm{ZFN}$ or other engineered nucleases, especially endogenous gene-modified cells, are somewhat phenotypically indistinguishable. Therefore, alternative protocols for screening or enriching mutated cells are needed.

To overcome the hurdle, we developed a new protocol for generating mutated porcine embryos using a transiently transfected episomal reporter-based enrichment system for cells with ZFN-induced mutations (Kim et al., 2011; Kim et al., 2013). With the enrichment system, mutation frequencies of $8.7 \%$ to $47 \%$ (11- to 17 -fold higher than frequencies in non-enriched cell populations) can be achieved. Moreover, gene-modified cells enriched with this system are alive and suitable for subsequent experiments. Hence, we assumed that this enrichment system, in combination with the SCNT technique, could provide a new generalized platform for producing mutated embryos. In the present study, we assessed the enrichment system in porcine cells and analyzed mutations in cloned pig embryos derived from the enriched ZFN-treated cells.

\section{MATERIALS AND METHODS}

\section{ZFNs and surrogate reporters}

Plasmids encoding ZFNs that target exon 6 of the pig CMP-N-acetylneuraminic acid hydroxylase (CMAH) gene were previously described (Kim et al., 2009). Enrichment system with reporters, eGFP or $\mathrm{H}-2 \mathrm{~K}^{\mathrm{k}}$ as selection markers for fluorescence activated cell sorting (FACS) and magnetic activated cell sorting (MACS), respectively were constructed as previously described (Kim et al., 2011; Kim et al., 2013).

\section{Preparation of cells and culture conditions}

A primary culture of pig fetal fibroblast cells that has been established as described by Cho et al. (2011) was used. Briefly, fetal tissues were minced and dissociated in TrypLE Express (Gibco, CA, USA) for $10 \mathrm{~min}$ at $37^{\circ} \mathrm{C}$. Cells were cultured in Dulbecco's modified Eagle's/Nutrient Mixture F-12 medium (DMEM/F12, Gibco) supplemented with 10\% (v/v) fetal bovine serum, $1 \mathrm{mM}$ Glutamax I (Gibco), $25 \mathrm{mM}$ $\mathrm{NaHCO}_{3}, 1 \%(\mathrm{v} / \mathrm{v})$ minimal essential medium, nonessential amino acid solution (Gibco) and $1 \%$ (v/v) Anti-Anti (Gibco) at $39^{\circ} \mathrm{C}$ in a humidified atmosphere of $5 \% \mathrm{CO}_{2}$ and $95 \%$ air.

\section{Plasmid DNAs transfection}

Fibroblasts were cultured to $80 \%$ to $90 \%$ confluence, then washed twice with D-PBS(-) (Gibco) and treated with $0.05 \%$ trypsin-EDTA (Gibco) to isolate and collect. Fibroblasts $\left(2 \times 10^{6}\right.$ cells $)$ were electroporated using a 100 $\mu \mathrm{L}$ Nucleocuvette, CA137 program code, in an Amaxa 4DNucleofector (Lonza, P3 Primary Cell 4D-Nucleofector X Kit L) with a total of $45 \mu \mathrm{g}$ plasmid DNA at a 2:2:1 weight ratio (plasmid encoding a left $\mathrm{ZFN}$ : plasmid encoding a right $\mathrm{ZFN}$ : eGFP reporter or $\mathrm{H}-2 \mathrm{~K}^{\mathrm{k}}$ reporter). Mutant cells were enriched using a flow cytometer (FACSAria III, BD Biosciences, USA) or MACSelect $\mathrm{K}^{\mathrm{k}}$ System (Miltenyi Biotec, Bergisch Gladbach, Germany) as described (Kim et al., 2011; Kim et al., 2013). The sorted cells were cultured for 2 additional days after sorting prior to SCNT or mutation analysis for proliferation and removing the dead cells.

\section{Analysis of mutations}

For detection of ZFN-induced mutations at the pig CMAH locus, the ZFN target locus was amplified from genomic DNA isolated from fibroblasts or cloned blastocysts (DNeasy kit, Qiagen) by nested PCR and subjected to the T7E1 assay (Kim et al., 2009). Primers used for the amplifications of the CMAH locus were as follows: 1st PCR/ 5'-tgtggacgtgccagactat-3' and 5'aaggcaatcaggctccttag- $3^{\prime}$, 2nd PCR/ 5'-tctacggaaatgctcctgct$3^{\prime}$ and $5^{\prime}$-tctacggaaatgctcctgct-3'.

For sequence analysis, PCR amplicons that included ZFN-target sites were purified using the Gel Extraction Kit (MACHERRY-ALGEN) and cloned into the T-Blunt vector using the T-Blunt PCR Cloning Kit (SolGent). Cloned plasmids were sequenced using the primers used for PCR amplification.

\section{Production and culture of cloned porcine embryos}

In vitro maturation of porcine oocytes, SCNT, and in vitro culture of the cloned embryos were performed as described elsewhere (Park et al., 2012) with slight modification. Briefly, ovaries were collected at a local abattoir and transported to the laboratory in sterile physical saline at $30^{\circ} \mathrm{C}$ to $35^{\circ} \mathrm{C}$. Cumulus-oocyte complexes (COCs) were aspirated from antral follicles (3 to $6 \mathrm{~mm}$ ) with 18 gauge needle attached to a $10 \mathrm{~mL}$ disposable syringe. COCs with several layers of cumulus cells and uniform cytoplasm were chosen and cultured in tissue culture medium 199 
(Gibco) supplemented with $10 \mathrm{ng} / \mathrm{mL}$ EGF, $0.57 \mathrm{mM}$ cysteine, $0.91 \mathrm{mM}$ sodium pyruvate, $5 \mu \mathrm{g} / \mathrm{mL}$ insulin, $1 \%$ (v/v) Pen-Strep (Gibco) and 10\% porcine follicular fluid at $39^{\circ} \mathrm{C}$ in a humidified atmosphere of $5 \% \mathrm{CO}_{2}$. For first $22 \mathrm{~h}$ of culture, $0.5 \mu \mathrm{g} / \mathrm{mL}$ follicle stimulating hormone and 0.5 $\mu \mathrm{g} / \mathrm{mL}$ luteinizing hormone were added to the culture medium and then removed for a further $22 \mathrm{~h}$. After a total of $44 \mathrm{~h}$ maturation culture, oocytes were denuded by pipetting with $0.1 \%$ hyaluronidase in TALP medium supplemented with $0.1 \%$ polyvinyl alcohol. Denuded oocytes with evenlygranulated and homogeneous cytoplasm were selected and then utilized for SCNT. A cumulus-free oocyte was held with a holding micropipette and the zona pellucida was partially dissected with a fine glass needle to make a slit near the adjacent cytoplasm, presumably containing the metaphase-II chromosomes, were extruded by aspiration with the same needle. Enucleation was confirmed by staining the cytoplasm with $0.5 \mu \mathrm{g} / \mathrm{mL}$ bisbenzimide during manipulation. ZFN-treated and sorted cells were trypsinized and observed under a microscope (Nikon). Cells with impaired membrane were excluded as morphological changes in cell membrane like irregular cell surface indicate cell death (Buja et al., 1993; Van Cruchten and Van Den Broeck, 2002). The cells expressed both RFP and eGFP (Figure 2a, circled) were manually selected using a micromanipulator (Nikon-Narishige, Japan) and fused with enucleated porcine oocytes (Figure 2b) with an electro cell fusion generator (LF101, Nepagene, Ichikawa, Japan) by applying a single direct current pulse $(200 \mathrm{~V} / \mathrm{mm}, 20 \mu \mathrm{s})$ and a pre- and post-pulse alterating current field of $5 \mathrm{~V}, 1$

(a)

\section{CMAH ExON 6}

ATCACGTACCTTACTCACGCCTGCATGGACCTCA AGCTGGGAGACAAGAGGATGGTGTTCGATCCTTG GTTAATCGGTCCTGCTTTTGCGCGAGGATGGTGG TTACTACACGAGCCTCCATCTGATTGGCTGGAGA GGCTGAGCCGCGCAGATTTAATTTACATCAGTCA CATGCACTCAGACCACCTGAG

(b)

\begin{tabular}{|c|c|c|c|c|}
\hline ZFN & $2 F 1$ & 252 & 253 & $1 F 4$ \\
\hline CM A H Left & QSHV & DSNRa & rdne & rdnq \\
\hline CMA H Bight & RDHT & RDHT & QSHR2 & QSHV \\
\hline
\end{tabular}

$\mathrm{MHz}$, for $5 \mathrm{~s}$, respectively. After 0.5 to $1.5 \mathrm{~h}$ oocytes were activated with a single DC pulse of $1.5 \mathrm{kV} / \mathrm{cm}$ for $60 \mu \mathrm{s}$ utilizing BTX electro-cell Manipulator 2001 (BTX, Inc., San Diego, USA). Reconstructed embryos were cultured in porcine zygote medium-5 (Funakoshi, IFP0410P, Tokyo, Japan) was maintained under a humidified atmosphere of $5 \% \mathrm{CO}_{2}, 5 \% \mathrm{O}_{2}$, and $90 \% \mathrm{~N}_{2}$ at $38.5^{\circ} \mathrm{C}$ for 7 days; cleavage and blastocyst rates were recorded at day 2 and 7 , respectively.

\section{Statistical analysis}

One way ANOVA analysis was performed to compare cleavages and blastocysts rates between each group after SCNT using Prism software (Version 6, GraphPad).

\section{RESULTS AND DISCUSSION}

Unlike in rodents, it is technically and economically difficult to obtain in vivo fertilized porcine embryos for genetic manipulation. Moreover, the high incidence of polyspermy (Gil et al., 2010) and low cryo-survival of boar sperm (Rodriguez-Martinez and Wallgren, 2010) also create problems for the production of in vitro fertilized embryos in this species. Therefore, microinjection of $\mathrm{ZFNs}$ into fertilized embryos, a technique used for producing knockout rodents, cannot easily be translated to pigs in most laboratories. On that account, we modified our SCNT protocols to produce mutated pig embryos. In first, mutated cells were prepared by transfection of ZFNs into porcine fibroblasts, coupled with reporter-based enrichment systems utilizing two different cell sorting methods, FACS and

(c)

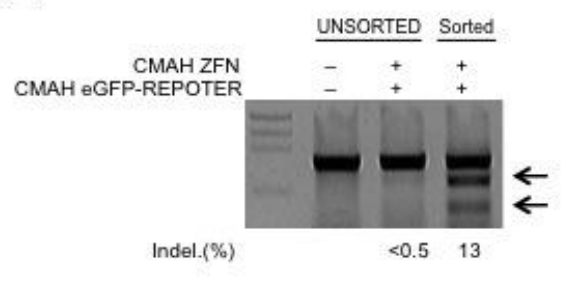

(d)

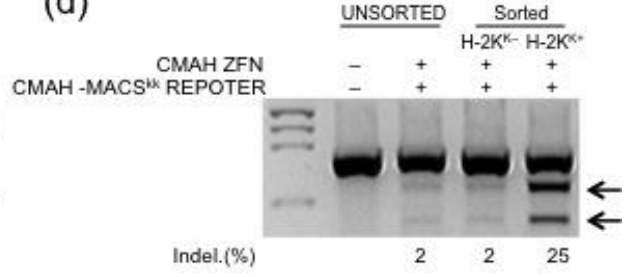

Figure 1. Enrichment system for cells containing ZFN-mediated mutations. (a) Sequences of the ZFN binding sites in the CMAH gene. The binding site of the right (red letters) and left (blue letters) ZFNs and the spacer sequence (underlined) are indicated. (b) The zinc finger module composition of CMAH ZFNs. (c and d) ZFN-driven CMAH mutations detected by the T7E1 assay in cell populations isolated by FACS (c) and MACS (d) using a ZFN surrogate reporter. H-2 $\mathrm{K}^{\mathrm{k}}$, a truncated mouse MHC class I, is used a selection marker for MACS. Arrows indicate the expected positions of DNA bands from specific cleavage of a mutated site by the mismatch-sensitive T7E1 enzyme. The numbers at the bottom of the gel indicate mutation percentages calculated by band intensities. 
(a)

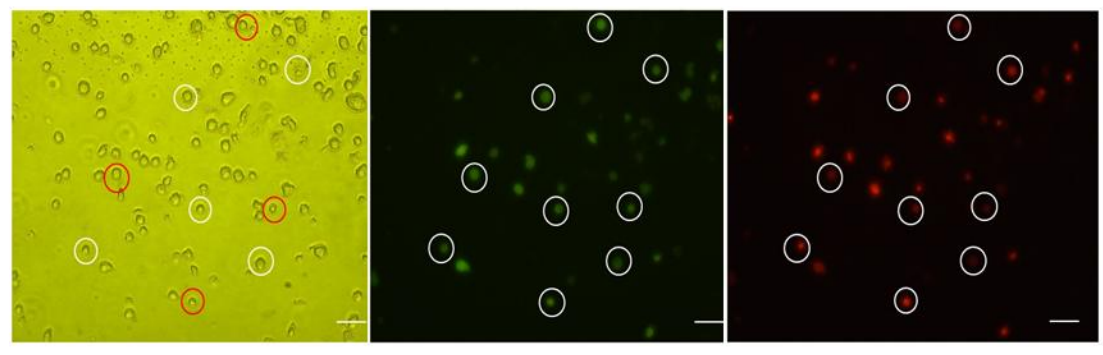

(b)

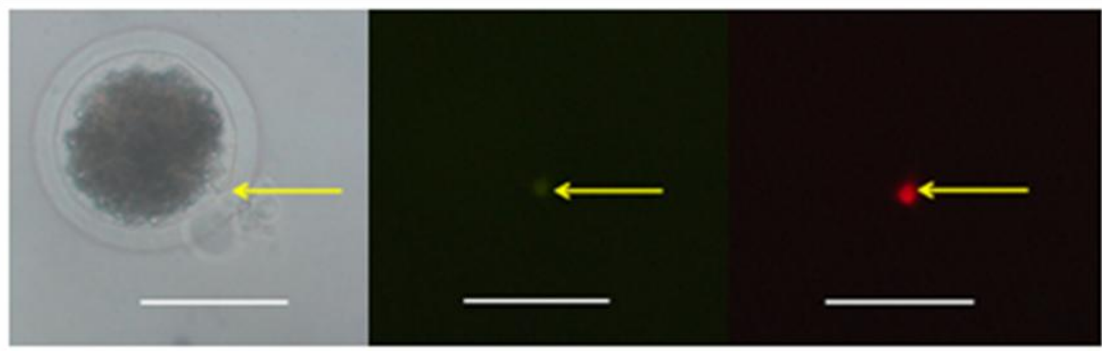

Figure 2. Somatic cell nuclear transfer with ZFN-treated donor cells. (a) Cells with intact membrane (circled in red) expressing both eGFP and RFP simultaneously (circled in white) were selected as donor cells (scale bar $=200 \mu \mathrm{m}$ ). (b) A manually-selected donor cell (arrow) was fused with a porcine oocyte matured in vitro (scale bar $=100 \mu \mathrm{m}$ ).

MACS, described previously (Kim et al., 2011; Kim et al., 2013). The enriched mutated cells were used as donor cells for SCNT. During the SCNT procedure, we re-confirmed the expression of reporter genes under microscope and only the cells with double positive for RFP and eGFP expressions (Figure 2a, circled) were used to produce cloned embryos.

To test the feasibility of using these knockout cell enrichment approaches for pig fibroblast cells, we used ZFNs targeting CMAH exon 6 (Figure 1a) and surrogate reporters containing either the eGFP gene alone (eGFP reporter) or eGFP and a truncated $\mathrm{H}-2 \mathrm{~K}^{\mathrm{k}}$ surface marker (MACS reporter), whose expression in cells can be recovered by a $\mathrm{ZFN}$-induced frameshift mutation in CMAH. Indeed, the use of both surrogate reporter systems together with appropriate sorting methods (FACS or MACS) successfully supported enrichment of fibroblasts containing ZFN-induced mutations in CMAH (Figure 1c and d). When these mutant-enriched cell populations were subjected to SCNT, the competence of reconstructed embryos derived from the MACS-separated and FACS-separated cell populations was comparable. Importantly, whereas the cloned embryos from the MACS-separated cell population
Table 1. In vitro culture of cloned embryos derived from ZFNtreated donor cells

\begin{tabular}{lccc}
\hline & $\mathrm{n}$ & Cleavage $(\%)$ & Blastocyst (\%) \\
\hline Control & 105 & $82(77.7 \pm 4.5)$ & $20(24.3 \pm 0.8)^{\mathrm{a}}$ \\
FACS & 157 & $116(61.11 \pm 6.5)$ & $16(13.1 \pm 2.5)^{\mathrm{b}}$ \\
MACS & 119 & $83(65.0 \pm 1.9)$ & $14(16.7 \pm 1.6)^{\mathrm{ab}}$ \\
\hline
\end{tabular}

${ }^{a, b}$ Values with different superscript letters in the same column indicate significant differences.

showed a similar rate of blastocyst development as compared to the control, the blastocyst development of the cloned embryos from the FACS-separated cell population was significantly impaired (Table $1 ; 13.1 \pm 2.5 \%$ vs $24.3 \pm$ $0.8 \%$ ). This difference might be caused by various stresses - including hydraulic pressure, shear force, vibrations, and high voltages — that cells experience during flow cytometry (Wolff et al., 2003). Consistent with this idea, large numbers of membrane-damaged cells were observed after FACS-mediated cell separation.

We also analyzed the ZFN target sites in the cloned blastocysts. The T7e1 assay results revealed that $\mathrm{ZFN}$ induced mutations at the target site in embryos from both the FACS and MACS groups (Figure 3a). Mutated (a)

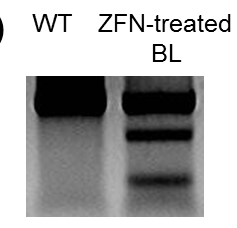

(b)

FACS

MACS

\begin{abstract}
ggtcctgcttttgcgcgaggatg
ggtcctgctt-----cgaggatg

ggtcctgctt国g国cgaggatg
\end{abstract}

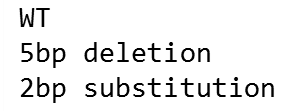

WT

4bp insertion

Figure 3. Mutations in cloned blastocysts derived from ZFN-treated cells. (a) The T7e1 assay showed ZFN-mediated mutations in cloned blastocysts. (b) Genomic DNA sequences from the cloned blastocyst. 
sequences, including insertions, deletions, and substitutions at the target site, as well as the wild-type sequence, were found in the assessed blastocysts (Figure $3 b$ ). Therefore, the mutations established in the study might not be biallelic. Interestingly, three different types of sequences were detected in the FACS group. This result revealed that the ZFN-initiated double-strand breaks and repair by the nonhomologous end joining pathways might be still ongoing after the one-cell embryo stage. This phenomenon has been commonly observed in various species of animals derived from ZFN-treated embryos, such as zebrafish (Doyon et al., 2008), mouse (Carbery et al., 2010), rabbit (Flisikowska et al., 2011), and rat (Geurts et al., 2009). Recently, mutant mice with biallelic deletions of the target site were obtained by injection of transcription activator-like effector nucleases (TALENs), another type of artificial restriction enzyme, into 1-cell embryos (Sung et al., 2013). Thus, the use of TALENs instead of ZFNs might facilitate the establishment of homozygous mutations in pig embryos. Our reporter based enrichment system also can be used with TALENs, therefore, it can be recommended to modify the current method for using TALENs instead of ZFNs to produce biallelic mutated pigs.

Recent reports showed that knockout pigs could be produced by co-transfection of nucleases (ZFNs or TALENs) with an antibiotic-selection marker followed by clonal selection (Yang et al., 2011; Carlson et al., 2012). These previous reports suggested a novel approach for generating knockout pigs. However, clonal selection from a single primary cell requires a lengthy period of time and sophisticated techniques. Use of the reporter-based enrichment system represents an alternative to the antibiotic-based selection method for reducing the time and effort needed to produce knockout pigs.

In conclusion, our results show that porcine transgenesis can be accomplished using ZFNs together with an enrichment reporter system. We described two types of transient reporter enrichment systems that can enrich cells with nuclease-induced mutation using FACS or MACS. The cloned embryos derived from cells enriched using MACS showed better developmental competence than did those derived from cells enriched by FACS. The desired mutated sequence was found in both systems. Therefore, the current procedure could provide a new generalized platform for producing mutated porcine embryos.

\section{ACKNOWLEDGEMENTS}

This study was supported by IPET (\#311011-05-3SB010), MKE (\#1033839-2013-21), Research Institute for Veterinary Science, BK21 program for Veterinary Science, and Cooperative Research Program for Agriculture Science \& Technology Development (Project No. PJ009802),
Biogreen21 (\#PJ009096), Rural Development Administration, Republic of Korea and Samsung Biomedical Research Institute (\#SA2013).

\section{REFERENCES}

Buja, L. M., M. L. Eigenbrodt, and E. H. Eigenbrodt. 1993. Apoptosis and necrosis. Basic types and mechanisms of cell death. Arch. Pathol. Lab. Med. 117:1208-1214.

Carbery, I. D., D. Ji, A. Harrington, V. Brown, E. J. Weinstein, L. Liaw, and X. Cui. 2010. Targeted genome modification in mice using zinc-finger nucleases. Genetics 186:451-459.

Carlson, D. F., W. Tan, S. G. Lillico, D. Stverakova, C. Proudfoot, M. Christian, D. F. Voytas, C. R. Long, C. B. Whitelaw, and S. C. Fahrenkrug. 2012. Efficient talen-mediated gene knockout in livestock. Proc. Natl. Acad. Sci. USA. 109:17382-17387.

Cho, B., O. J. Koo, J. I. Hwang, H. Kim, E. M. Lee, S. Hurh, S. J. Park, H. Ro, J. Yang, C. D. Surh, A. J. D'Apice, B. C. Lee, and C. Ahn. 2011. Generation of soluble human tumor necrosis factor-alpha receptor $1-\mathrm{fc}$ transgenic pig. Transplantation 92:139-147.

Doyon, Y., J. M. McCammon, J. C. Miller, F. Faraji, C. Ngo, G. E. Katibah, R. Amora, T. D. Hocking, L. Zhang, E. J. Rebar, P. D. Gregory, F. D. Unnov, and S. L. Amacher. 2008. Heritable targeted gene disruption in zebrafish using designed zincfinger nucleases. Nat Biotechnol. 26:702-708.

Flisikowska, T., I. S. Thorey, S. Offner, F. Ros, V. Lifke, B. Zeitler, O. Rottmann, A. Vincent, L. Zhang, S. Jenkins, H. Niersbach, A. J. Kind, P. D. Gregory, A. E. Schnieke, and J. Platzer. 2011. Efficient immunoglobulin gene disruption and targeted replacement in rabbit using zinc finger nucleases. PLoS One. 6(6): 21045.

Geurts, A. M., G. J. Cost, Y. Freyvert, B. Zeitler, J. C. Miller, V. M. Choi, S. S. Jenkins, A. Wood, X. Cui, X. Meng, A. Vincent, S. Lam, M. Michalkiewicz, R. Schilling, J. Foeckler, S. Kalloway, H. Weiler, S. Menoret, I. Anegon, G. D. Davis, L. Zhang, E. J. Rebar, P. D. Gregory, F. D. Urnov, H. J. Jacob, and R. Buelow. 2009. Knockout rats via embryo microinjection of zinc-finger nucleases. Science 325(5939):433.

Gil, M. A., C. Cuello, I. Parrilla, J. M. Vazquez, J. Roca, and E. A. Martinez. 2010. Advances in swine in vitro embryo production technologies. Reprod. Domest. Anim. 45 (Suppl 2):40-48.

Hauschild, J., B. Petersen, Y. Santiago, A. L. Queisser, J. W. Carnwath, A. Lucas-Hahn, L. Zhang, X. Meng, P. D. Gregory, R. Schwinzer, G. J. Cost, and H. Niemann. 2011. Efficient generation of a biallelic knockout in pigs using zinc-finger nucleases. Proc. Natl. Acad. Sci. USA. 108:12013-12017.

Kim, H., M. S. Kim, G. Wee, C. I. Lee, H. Kim, and J. S. Kim. 2013. Magnetic separation and antibiotics selection enable enrichment of cells with zfn/talen-induced mutations. PLoS One. 8(2):e56476.

Kim, H., E. Um, S. R. Cho, C. Jung, H. Kim, and J. S. Kim. 2011. Surrogate reporters for enrichment of cells with nucleaseinduced mutations. Nat. Methods 8:941-943.

Kim, H. J., H. J. Lee, H. Kim, S. W. Cho, and J. S. Kim. 2009. Targeted genome editing in human cells with zinc finger nucleases constructed via modular assembly. Genome Res. 19:1279-1288. 
Kim, S., M. J. Lee, H. Kim, M. Kang, and J. S. Kim. 2011. Preassembled zinc-finger arrays for rapid construction of zfns. Nat. Methods 8:7-9.

Koo, O. J., G. Jang, and B. C. Lee. 2012. Minipig as laboratory animals: Facility management and husbandry. Reprod. Dev. Biol. 36:79-85.

Li, P., J. L. Estrada, C. Burlak, and A. J. Tector. 2013. Biallelic knockout of the alpha-1,3 galactosyltransferase gene in porcine liver-derived cells using zinc finger nucleases. J. Surg. Res. 181:e39-45.

Lutz, A. J., P. Li, J. L. Estrada, R. A. Sidner, R. K. Chihara, S. M. Downey, C. Burlak, Z. Y. Wang, L. M. Reyes, B. Ivary, F. Yin, R. L. Blankenship, L. L. Paris, and A. J. Tector. 2013. Double knockout pigs deficient in n-glycolylneuraminic acid and galactose alpha-1,3-galactose reduce the humoral barrier to xenotransplantation. Xenotransplantation 20:27-35.

Park, S. J., H. J. Park, O. J. Koo, W. J. Choi, J. H. Moon, D. K. Kwon, J. T. Kang, S. Kim, J. Y. Choi, G. Jang, and B. C. Lee. 2012. Oxamflatin improves developmental competence of porcine somatic cell nuclear transfer embryos. Cell. Reprogram. 14:398-406.

Rodriguez-Martinez, H. and M. Wallgren. 2011. Advances in boar semen cryopreservation. Vet. Med. Int. Vol. 2011, Article ID 396181, 5 pages, doi:10.4061/2011/396181.

Smith, A. C. and M. M. Swindle. 2006. Preparation of swine for the laboratory. ILAR J. 47:358-363.
Sung, Y. H., I. J. Baek, D. H. Kim, J. Jeon, J. Lee, K. Lee, D. Jeong, J. S. Kim, and H. W. Lee. 2013. Knockout mice created by talen-mediated gene targeting. Nat. Biotechnol. 31:23-24.

Van Cruchten, S. and W. Van Den Broeck. 2002. Morphological and biochemical aspects of apoptosis, oncosis and necrosis. Anat. Histol. Embryol. 31:214-223.

Watanabe, M., K. Umeyama, H. Matsunari, S. Takayanagi, E. Haruyama, K. Nakano, T. Fujiwara, Y. Ikezawa, H. Nakauchi, and H. Nagashima. 2010. Knockout of exogenous egfp gene in porcine somatic cells using zinc-finger nucleases. Biochem. Biophys. Res. Commun. 402:14-18.

Whyte, J. J. and R. S. Prather. 2011. Genetic modifications of pigs for medicine and agriculture. Mol. Reprod. Dev. 78:879-891.

Whyte, J. J., J. Zhao, K. D. Wells, M. S. Samuel, K. M. Whitworth, E. M. Walters, M. H. Laughlin, and R. S. Prather. 2011. Gene targeting with zinc finger nucleases to produce cloned eGFP knockout pigs. Mol. Reprod. Dev. 78:2.

Wolff, A., I. R. Perch-Nielsen, U. D. Larsen, P. Friis, G. Goranovic, C. R. Poulsen, J. P. Kutter, and P. Telleman. 2003. Integrating advanced functionality in a microfabricated high-throughput fluorescent-activated cell sorter. Lab Chip. 3:22-27.

Yang, D., H. Yang, W. Li, B. Zhao, Z. Ouyang, Z. Liu, Y. Zhao, N. Fan, J. Song, J. Tian, F. Li, J. Zhang, L. Chang, D. Pei, Y. E. Chen, and L. Lai. 2011. Generation of PPARgamma monoallelic knockout pigs via zinc-finger nucleases and nuclear transfer cloning. Cell Res. 21:979-982. 